\title{
RESEARCH AND DEVELOPMENT OF THE ANTENNA ARRAY FOR GROUND PENETRATING RADAR
}

\author{
Evgeny Balzovsky ${ }^{*}$, Yury Buyanov \\ National Research Tomsk State University, 634050, Tomsk, Russia
}

\begin{abstract}
The design and results of investigation of the antenna array for ground penetrating radar are presented. The antenna array is intended for measurement of the electrical properties of the road coating and finding of irregularities at monitoring the quality of roads. Antenna elements are made by PCB technology on the plate of FR4. Each antenna element is placed in a reflector formed by aluminum corner. The results of simulation in CST Studio Suite and the results of measurements of the characteristics of the antenna elements, and their mutual influence are presented. The developed antenna array can be used at the ultrawideband pulse excitation, and at a scanning of continuous frequencies in the range of $0.5-2 \mathrm{GHz}$.
\end{abstract}

\section{Introduction}

The ground penetrating radar (GPR) technology is promising for the study of the quality of roads and finding of a latent defects in the asphalt coating during the construction and maintenance of roads to prevent damage to the roadway [1]. Monitoring the status of the roadway portion includes determining the thickness and homogeneity of the top coat layer consisting of a asphalt concrete mix, thickness and humidity of the underlying layers of gravel or crushed stone as well as the detection and localization of cavities [2-4].

Despite the fact that there are a number of technical solutions, as well as the finished commercial devices for sensing of the roadway, a significant obstacle to the widespread introduction of such devices in the practice of diagnosing the state of any roads is low scanning speed while moving the scanner on long stretches of road, as well as the high cost of such devices.

The technologies and algorithms developed for radio-wave imaging systems of objects hidden behind the optically nontransparent obstacles [4] can be applied to create a new inexpensive scanner for inspection of quality of highway coating. Irradiation of the surface layer of asphalt concrete pavement and the underlying layers of pavement by ultrawideband pulses of nanosecond duration provides both the high spatial resolution and the deep penetration of electromagnetic waves due to availability of energy in the low frequency part of the pulse spectrum.

* Corresponding author: tduty5@mail.ru 
For construction of the radar image of the layered structure and the irregularities distribution of the roadway the transceiver of the radar should move along the traffic lane to use the method of synthetic aperture. To increase the width of the scanned lane is advantageous to use switched matrices of transmitting and receiving ultrawideband antennas. Due to the complexity of beam-forming network of the phased antenna array and a high cost of its components it is advantageous to use a switched antenna array. In such matrix the antennas are sequentially connect to the generator or receiver [5], and beamforming algorithm is performed with mathematical processing in a computer.

For the effective radiation of the ultrawideband signals and to record the reflected radiation the antenna should be matched with the feeder in the frequency band of two or more octaves. The pattern of the antenna should have a constant main lobe direction in the same frequency band. Moreover, the antenna should have the smallest possible size if it is used as an element of the array. The antennas that are a combination of electric type and magnetic type radiators [6] satisfy these requirements.

The high spatial resolution can be achieved by the ultrawideband pulses with the spectrum occupying a bandwidth 1-5 GHz. However, the losses in the probed medium increase with increasing of frequency, so to increasing the probing depth it is necessary to use the signals with the spectrum that is shifted toward lower frequencies. The design of the planar ultrawideband antenna with a bandwidth of $0.5-2 \mathrm{GHz}$ is described below. The results of numerical simulation and the measured characteristics are presented.

\section{The design of antenna array}

The embodiment of the antenna array is shown in Figure 1. The antenna elements 1 are divided into the modules of four antennas and arranged in an aluminum corner 2 . The distance between the antennas is equal to $150 \mathrm{~mm}$. The length of one module is equal to $0.6 \mathrm{~m}$. The number of modules depends on the application and can be arbitrary. The number of blocks determines the width of scanned strip of road surface. The antenna array is connected to the switching unit via coaxial connectors 3 . The whole array is covered with a plastic housing and mounted on a mobile platform.

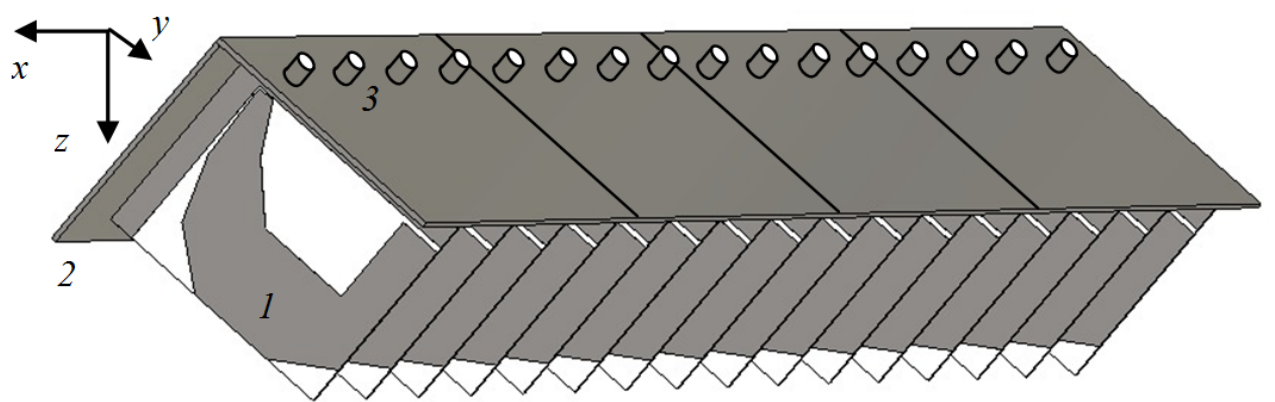

Figure 1. The design of the antenna array for GPR: 1 - antenna elements, 2 - aluminum corner as a reflector, 3 - coaxial connectors.

\section{The antenna element}

\subsection{Design of the antenna element}

The developed antenna is made on a plate of FR4 with size of $110 \times 110 \times 1 \mathrm{~mm}$. The electric monopole 1 in Figure 2 is made by PCB technology. The area of the plate 2 that is left free 
from metal foil represents a radiator of magnetic type. The plate is mounted in the aluminum corner 3 with cross-section of $120 \times 120 \mathrm{~mm}$.

The necessary amplitude-phase relations for the excitation both types of radiators are defined by the sizes of the area 2 and a slot line 4 . Tapered slot line 4 performs the function of the impedance transformer. On the back side of the corner the coaxial connector is mounted. The central electrode of the connector is connected to the monopole in the point 5. The direction of main lobe of antenna pattern is indicated by the arrow.

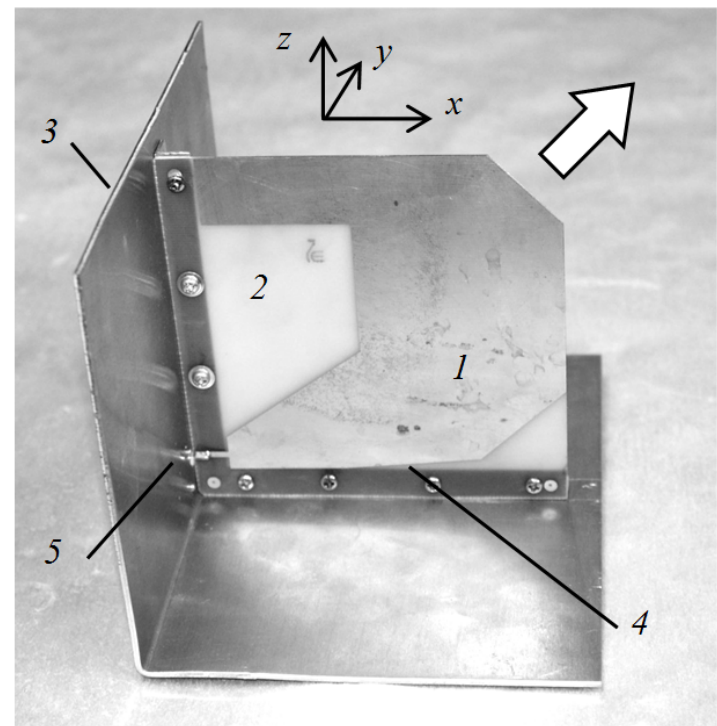

Figure 2. The design of antenna element: 1 - electrical monopole, $2^{-}$- magnetic type radiator, 3 - aluminum corner as a reflector, 4 - tapered slot line, 5 - coaxial connector.

\subsection{Characteristics of the antenna element}

Modeling of antenna element was performed in electromagnetic field simulation software SCT Studio Suite under Educational License. The results of calculation of the voltage stand-wave ratio (VSWR) of a single antenna element in are shown in Figure 3 (curve 1). In the same Figure (curve 2) the VSWR measured by network analyzer Agilent N5230C is shown. There is a good enough agreement between calculated and measured VSWR. The difference of curves may be due to the fact that the losses in the dielectric and the metal had not been taken into account in the model.

The direction of the main lobe of antenna pattern $\left(\theta_{0}, \varphi_{0}\right)$ lies in the $x z$-plane and is directed at an angle $\theta_{0}=45$ degrees to the $z$-axis over a wide frequency range. The radiated electric field has a linear polarization in the main lobe direction. The plane of polarization coincides with the plane of PCB plate of the antenna. The calculated patterns of the antenna element in $E$-plane and $H$-plane are shown in Figure 4 and Figure 5 (curves 1 ). The origin (angle $=0)$ matches the direction of $\left(\theta_{0}, \varphi_{0}\right)$.

Measurements of antenna patterns were held in the time domain at the excitation of the antenna by bipolar voltage pulses of duration of 0.5 and $1 \mathrm{~ns}$. For each angular position of the antenna under study the pulse waveform at the output of the auxiliary receiving antenna was recorded by the sampling oscilloscope. An angular dependence of the spectral components in the range of $0.5 \ldots 5 \mathrm{GHz}$ was obtained using Fourier transform. The rectangular temporal window was used for filtering signals reflected from the surrounding 
environment. The measured patterns of the antenna element at both $E$ - and $H$-planes are presented in Figure 4 and Figure 5 (curves 2).

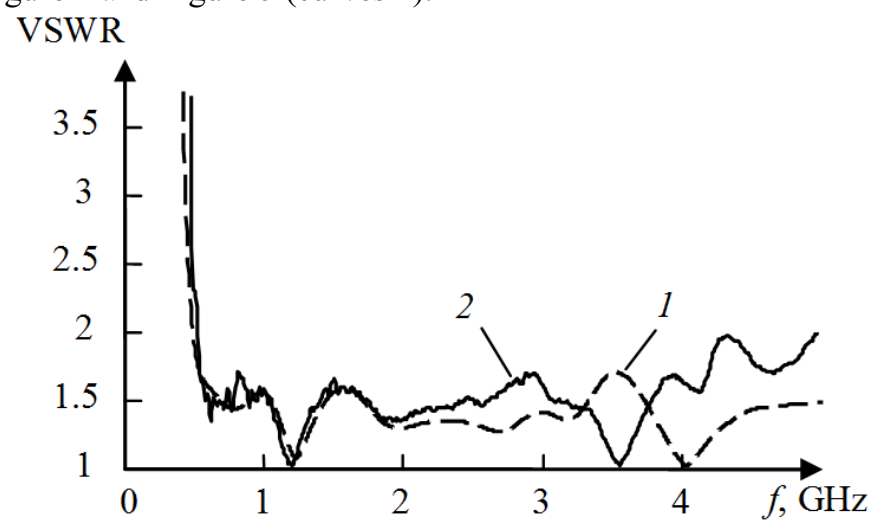

Figure 3. VSWR of the antenna element: 1 - simulations, 2 - measurements.
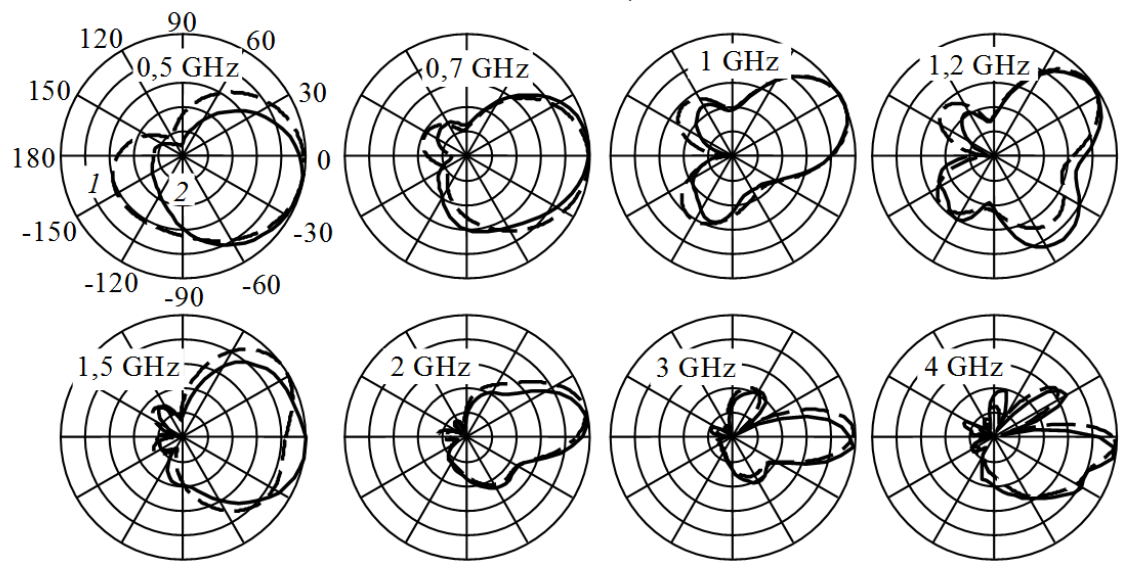

Figure 4. Patterns of the antenna element at the $E$-plane.
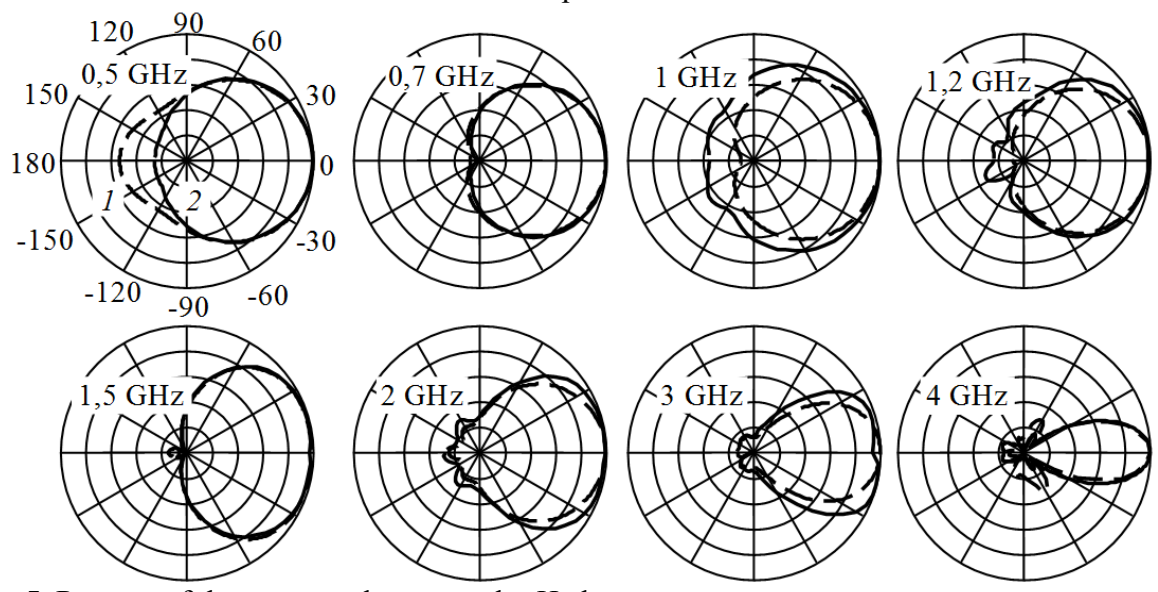

Figure 5. Patterns of the antenna element at the $H$-plane. 


\section{Characteristics of the antenna array}

An appearance of one module of the array antenna unit is shown in Figure 6. Since the antenna elements are located in close proximity to each other, it is necessary to consider their mutual influence. To estimate the mutual influence of the elements the measurements of VSWR were performed separately for external elements 1 and 4 in Figure 6 and for inner elements 2 and 3. The measurement results are shown in Figure 7. Change the length of the metal reflector and the presence of neighboring elements for external antennas 1 and 4 results in a slight shift of the lower boundaries of the band matching. At the rest of the frequency band VSWR does not exceed the value of 2. For internal elements ( 2 and 3 in Figure 6) was found the more significant shift of the lower limit of the bandwidth toward higher frequencies compared with a single antenna (curve 3 in Figure 7). Notwithstanding, the proportion of the reflected power in the frequency band of $0.5 \ldots 5 \mathrm{GHz}$ is not more than 0.2 of incident power.

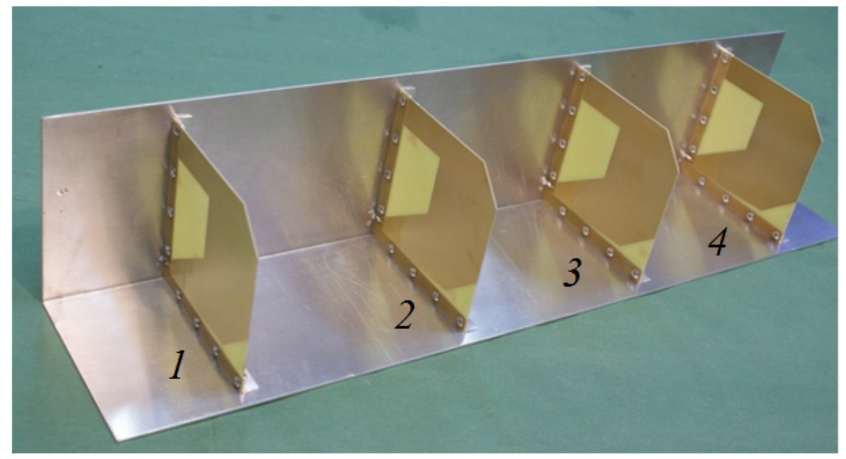

Figure 6. The design of four-element module of the antenna array.

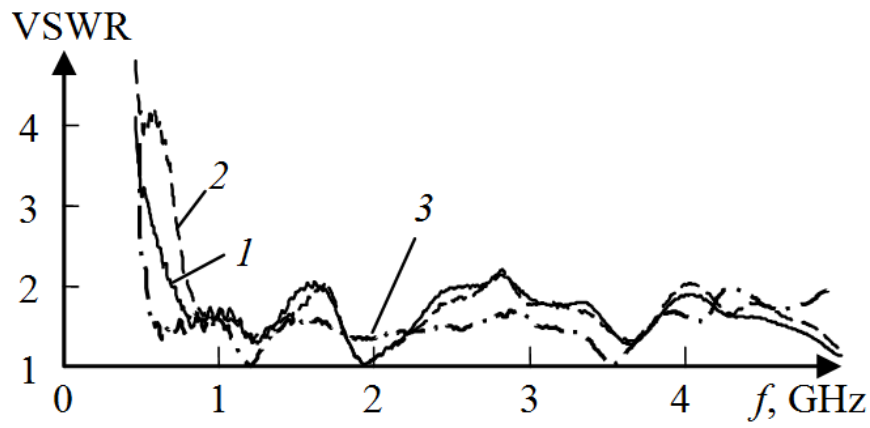

Figure 7. VSWR of antennas in the array. 1 - external antenna elements, 2 - internal antenna elements, 3 - single antenna.

The measured transmission coefficients $\left|S_{m n}\right|$ between antenna elements $n$ and $m$ (n, $m=1 \ldots 4)$ of the array are presented in Figure 8. The adjacent elements (pairs 1-2 and 2-3) have the greatest influence on each other. The maximum of $\left|S_{21}\right|$ and $\left|S_{32}\right|$ is located below the lower border of frequency band. The mutual influence of the distant elements is smaller than the neighboring. Further, in order to reduce the mutual influence of the antenna element it is assumed to fill partially the space between the antennas by a volume absorbing material. 


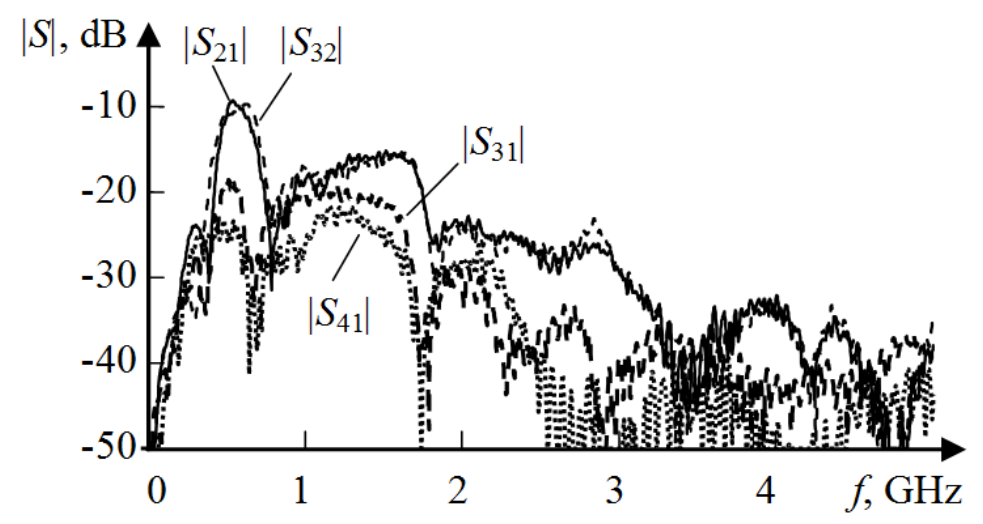

Figure 8. Transmission coefficients between antenna elements in array.

\section{Conclusion}

The ultrawideband antenna array for use in ground penetrating radar is developed and investigated. The antenna elements are matched with the 50 -ohm feeder in the frequency band of $0.5-5 \mathrm{GHz}$ and have a stable direction of main lobe of the pattern. Despite the close proximity of the elements in the array, the level of mutual interference elements is not critical, although it limiting the matching band at low frequencies. The developed array can be excited both by the ultrawideband short pulses and by the continuous scanning frequencies in the range of $0.5 \ldots 2 \mathrm{GHz}$.

\section{Acknowledgment}

This research is conducted as part of the Program of Scientific Foundation n. a. D.I. Mendeleev in Tomsk State University (Project No. 8.2.48.2015).

\section{References}

[1] D. Daniels Ground-penetrating radar (London, The Institution of Electrical Engineers, 2004)

[2] Z. Mechbal, A. Khamlichi, MATEC Web of Conferences 16, 07005 (2014) doi : 10.1051/matecconf/20141607005

[3] Guo M. L., Huang S. P., MATEC Web of Conferences 44, 02079 (2016) doi : $10.1051 /$ matecconf $/ 20164402079$

[4] V. P. Yakubov, S. E. Shipilov, D. Y. Sukhanov, A. V. Klokov Radiovolnovaya tomografiya: dostijeniya I perspektivi (Tomsk, NTL, 2014)

[5] R. N. Satarov, I. Y. Kuzmenko, T. R. Muksunov, A. V. Klokov, E. V. Balzovskii, Y. I. Buyanov, S. E. Shipilov, V. P. Yakubov, Rus. Phys. J. 55, 884 (2013) doi : 10.1007/s11182-013-9896-8

[6] Yu. A. Andreev, Yu. I. Buyanov, V. I. Koshelev, J. Commun. Technol. Electron. 50 , $535(2005)$ 